\section{Factores asociados al deterioro cognitivo en personas mayores en Chile}

\author{
MARÍA ADELA MARTÍNEZ-SANGUINETTI ${ }^{1, \mathrm{a}^{*} \text {, }}$ \\ ANA MARÍA LEIVA ${ }^{2, b^{*}}$, FANNY PETERMANN-ROCHA ${ }^{3,10, c}$, \\ CLAUDIA TRONCOSO-PANTOJA ${ }^{4, \mathrm{~d}}$, MARCELO VILLAGRÁN ${ }^{5, \mathrm{e}}$, \\ FABIÁN LANUZA-RILLING ${ }^{6, \mathrm{c}}$, GABRIELA NAZAR $^{7, \mathrm{f}}$, \\ FELIPE POBLETE-VALDERRAMA ${ }^{8, g}$, XIMENA DÍAZ-MARTÍNEZ $^{9, \mathrm{~h}}$, \\ CARLOS CELIS-MORALES ${ }^{10,11, \mathrm{i}}$ (En representación de todos
} los integrantes del grupo ELHOC-Epidemiology of Lifestyle and Health Outcomes in Chile)

\section{Factors associated with cognitive impairment in older adults in Chile}

Background: The risk factors for the development of cognitive impairment are not well known. Aim: To identify socio-demographic, lifestyle and health-related factors associated with cognitive impairment in older Chilean adults. Material and Methods: Data analysis of 1,384 participants $\geq 60$ years who participated in the National Health Survey of Chile 2009-2010. Sociodemographic, lifestyle and health-related factors were used as exposure variables of interest. Cognitive impairment was assessed using an abbreviated version of the Mini Mental test and defined as a score $<13$ points of a maximum of 19. A logistic regression was used to identify factors associated with cognitive impairment. Results: In this sample, the prevalence of cognitive impairment was 11.6 [95\% confidence intervals (CI): 8.8; 15.2]. The factors associated with cognitive impairment were age (Odds ratio $(\mathrm{OR})$ for $>76$ years: 4.89 , $p<0.01$ ), male sex (OR: 2.42, $p=0.02$ ), lower education (OR: 21.6, $p<0.01)$, physical inactivity (OR: 2.07, $p=0.02)$, sedentary behavior (OR: 2.23, $p=0.01$ ), sleeping $>$ 9/hours/day (OR: 2.98, $p=0.01$ ), consumption of $<5$ portions/day of fruit and vegetables (OR: 2.02, $p=0.05)$, having an unhealthy lifestyle (OR: $6.10, p=0.0001)$, being underweight (OR: 3.67, $p<0.01)$, obesity (OR: 3.32, $p=0.03)$, having hearing impairment (OR: 2.26, $p=0.02$ ), having a visual impairment (OR: 3.89, $p<0.01$ ), a history of depression (OR: 3.03, $p=0.01)$ and having a physical disability (OR: 5.63, $p<0.01$ ). Conclusions: We identified 14 factors associated with cognitive impairment. Although some of these factors were non-modifiable such as age and sex, most of them could be modified by implementing prevention programs aiming to improve lifestyle behaviors in older adults in Chile.

(Rev Med Chile 2019; 147: 1013-1023)

Key words: Aged; Cognitive Dysfunction; Life Style; Multimorbidity.
'Instituto de Farmacia, Facultad de Ciencias, Universidad Austral de Chile, Valdivia, Chile. 2Instituto de Anatomía, Histología y Patología, Facultad de Medicina, Universidad Austral de Chile. Valdivia, Chile.

${ }^{3}$ Institute of Health and Wellbeing, University of Glasgow. Glasgow, United Kingdom. ${ }^{4}$ CIEDE-UCSC, Departamento de Salud Pública, Facultad de Medicina, Universidad Católica de la Santísima Concepción.

Concepción, Chile.

${ }^{5}$ Departamento de Ciencias Básicas. Facultad de Medicina. Universidad Católica de la Santísima Concepción. Concepción, Chile. ${ }^{6}$ Departamento de Pediatría y Cirugía infantil. Facultad de Medicina. Universidad de La Frontera. Temuco, Chile.

${ }^{7}$ Departamento de Psicología y Centro de Vida Saludable. Facultad de Ciencias Sociales. Universidad de Concepción.

Concepción, Chile.

${ }^{8}$ Escuela de Kinesiología, Facultad de Salud, Universidad Santo Tomás, Sede Valdivia. Valdivia, Chile.

${ }^{9}$ Grupo de Investigación en Calidad de Vida, Departamento de Ciencias de la Educación, Facultad de Educación y Humanidades, Universidad del Biobío. Chillán, Chile. ${ }^{10}$ BHF Glasgow Cardiovascular Research Centre, Institute of Cardiovascular and Medical Science, University of Glasgow. Glasgow, United Kingdom.

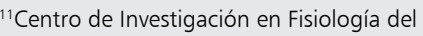
Ejercicio-CIFE, Universidad Mayor. Santiago, Chile.

aMSc Nutrición y Dietética.

${ }^{b}$ MSc. Neurociencias y Salud Mental.

"MSc Nutrición Humana.

${ }^{\mathrm{MSC}}$ Salud Pública basada en la evidencia; MSc Gerontología.

eDr. Ciencias Biológicas.

fDr. Psicología.

${ }^{9}$ MSc Educación en Salud y Bienestar

Humano

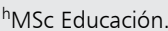

'PhD Ciencias Cardiovasculares y Biomédicas.

*MAMS y AML contribuyeron de igual manera en este manuscrito y deben ser consideradas primer autor compartido.

Trabajo no recibió financiamiento. Los autores declaran no tener conflictos de interés.

Recibido el 8 de enero de 2019, aceptado el 8 de agosto de 2019 .

Correspondencia a:

María Adela Martínez-Sanguinetti Instituto de Farmacia, Facultad de Ciencias, Universidad Austral de Chile, Valdivia, Chile. mmartin3@uach.cl 
T anto a nivel mundial como en Chile el envejecimiento se ha convertido en un área prioritaria en la planificación sanitaria ${ }^{1-6}$, no solo por el aumento de personas mayores, sino que, además por el aumento de multimorbilidad y deterioro cognitivo ${ }^{1-6}$. Si bien el envejecimiento se asocia a importantes cambios en nuestra salud, el deterioro cognitivo ha recibido un énfasis especial debido a su fuerte asociación con demencia ${ }^{1,3,6}$.

Existe una vasta cantidad de evidencia a nivel internacional que ha identificado factores de riesgo asociados a deterioro $\operatorname{cognitivo}{ }^{3,6,7}$, esta evidencia ha sido generada en su mayoría en países desarrollados, contando hoy en día con limitada evidencia proveniente de países en vías de desarrollo ${ }^{1,3,6,7}$. Según las nuevas directrices publicadas este año por la Organización Mundial de la Salud (OMS), practicar ejercicio físico de forma regular, no fumar, evitar el uso nocivo del alcohol, controlar el peso corporal, seguir una dieta sana y mantener la presión arterial, glicemia y el colesterol en niveles adecuados puede reducir el riesgo de padecer deterioro cognitivo y demencia ${ }^{1,5,6}$. Sin embargo, la mayor parte de la evidencia que respalda estas guías de la OMS se basa en estudios internacionales que podrían no representar la realidad de países latino americanos, entre ellos, Chile. Si bien nuestro país ha migrado a la lista de países de alto ingreso económico, su perfil epidemiológico caracteriza a una economía en etapa de transición debido a la alta prevalencia de obesidad, inactividad física, tabaquismo, alimentación no saludable y alta prevalencia de factores de riesgo cardiovascular incluyendo la diabetes e hipertensión ${ }^{8-10}$. Considerando el incremento en la expectativa de vida y consecuente incremento del número de personas mayores ${ }^{6,11}$ es crucial determinar si factores de riesgo para deterioro cognitivo que han sido identificado en estudios internacionales se asocian a sospecha de deterioro cognitivo en población adulta mayor en Chile. A la fecha existe una limitada cantidad de evidencia ${ }^{4,9,12-15}$, lo cual limita la creación de nuevas políticas públicas, intervenciones o programas de prevención orientados a disminuir aquellos factores de riesgo asociados a deterioro cognitivo en Chile. Considerando estos antecedentes, el objetivo de este estudio fue identificar qué factores sociodemográficos, de estilos de vida y salud se asocian a sospecha de deterioro cognitivo en personas mayores en Chile.

\section{Material y Método}

\section{Diseño del estudio}

Este estudio de corte transversal se basa en la Encuesta Nacional de Salud realizada entre los años 2009 y 2010 (ENS 2009-2010) ${ }^{10}$. De un total de 5.293 participantes encuestados en la ENS, 1.390 eran personas mayores (edad $>60$ años) y de estos solo 1.384 participantes tenían información disponible para la prueba de Mini Mental State Examination (MMSE), los cuales fueron incluidos en este estudio. Los protocolos de evaluación y recolección de datos de la ENS 2009-2010 fueron aprobados por el Comité de Ética de la Pontificia Universidad Católica de Chile. Todos los participantes firmaron un consentimiento informado previo a la recolección de datos ${ }^{10}$.

Para determinar sospecha de deterioro cognitivo se utilizó el cuestionario MMSE, versión abreviada, con 6 preguntas y puntaje máximo de 19 puntos; considerándose "sospecha de deterioro cognitivo" aquellos individuos con un puntaje $<13$ puntos $^{10}$.

Factores sociodemográficos tales como edad, sexo, zona de residencia (rural o urbana), nivel educacional $(<8,8-12$ y $>12$ años $)$ e ingreso económico (bajo, medio y alto), fueron autorreportados. Estilos de vida (alimentación, consumo de alcohol, actividad física y tabaquismo) fueron medidos mediante la aplicación de cuestionarios validados y descritos en la ENS 2009-2010 ${ }^{10}$. El consumo de sal se determinó mediante análisis de excreción de sodio en orina ${ }^{10}$. El consumo diario de alcohol fue categorizado según el puntaje AUDIT para consumo de alcohol ${ }^{10}$.

El tiempo destinado a dormir fue categorizado en base a recomendaciones internacionales $<7 \mathrm{~h} /$ día, 7-9 h/día y $>9$ h/día ${ }^{10}$. El tiempo destinado a actividad física (AF) durante el tiempo libre y/o en el trabajo se determinó con la guía de análisis de GPAQ (Global Physical Activity Questionnaire v2 $)^{10}$. La AF total fue expresada en MET/h/ semana. Se consideró inactividad física un gasto energético $<600 \mathrm{METs} / \mathrm{min} / \mathrm{semana}^{10}$. El tiempo sedente se determinó mediante el autorreporte del tiempo destinado a actividades que involucren estar sentado o reclinado durante el tiempo libre o de trabajo, clasificando a los participantes en dos niveles de sedentarismo; alto $(\geq 4 \mathrm{~h} /$ día $)$ y bajo ( $<4 \mathrm{~h} /$ día $)$, en base a estudios realizados en población nacional ${ }^{16}$. 
Se generó un índice de estilo de vida saludable ${ }^{9}$, el cual incluyó 7 componentes: i) consumo de 5 porciones de frutas y verduras al día; ii) consumo de sal $<8 \mathrm{~g} /$ día; iii) consumo de alcohol (puntaje test AUDIT $<8^{14}$; iv) dormir entre 7-9 h/día; v) nunca haber fumado; vi) ser físicamente activo ( $>150 \mathrm{~min} / \mathrm{semana}$ de AF moderada) y vii) tiempo sedentario $<4 \mathrm{~h} /$ día. Se asignó 1 punto por cada conducta saludable, con un puntaje máximo de 7 puntos, que correspondió al cumplimiento de todas las conductas indicadas. Para propósitos de este estudio, se clasificó el puntaje de estilos de vida en tertiles: no saludable: $0-1$ puntos; moderadamente saludable: $2-4$ puntos y muy saludable: 5-7 puntos, como ha sido reportado previamente ${ }^{9}$.

El estado nutricional fue determinado a través del índice de masa corporal (IMC: $\mathrm{kg} / \mathrm{m}^{2}$ ) y clasificado según los puntos de corte específicos para personas mayores (bajo peso: $<22,9 \mathrm{~kg} / \mathrm{m}^{2}$; normopeso: $23,0-27,9 \mathrm{~kg} / \mathrm{m}^{2}$; sobrepeso: $28,0-$ $31,9 \mathrm{~kg} / \mathrm{m}^{2}$ y obesidad: $\left.\geq 32,0 \mathrm{~kg} / \mathrm{m}^{2}\right)^{10}$. La obesidad central fue definida midiendo el perímetro de cintura, utilizando los puntos de corte sugeridos por la ENS 2009-2010 (mujeres $\geq 88 \mathrm{~cm}$ y hombres $\geq 102 \mathrm{~cm})^{10}$.

Marcadores metabólicos (glicemia basal, colesterol HDL y triglicéridos) fueron medidos en ayuno con métodos estandarizados y previamente descritos en la ENS 2009-2010 ${ }^{10}$. Para la detección de diabetes mellitus tipo 2, se consideró una glicemia en ayuno $>126 \mathrm{mg} / \mathrm{dl}$ o el autorreporte de diagnóstico médico de diabetes. La hipertensión arterial fue definida con tres mediciones $\geq 140 / 90$ $\mathrm{mmHg}$ o autorreporte de tratamiento farmacológico para hipertensión. Se consideró síndrome metabólico a la presencia de 3 o más de los 5 criterios definidos en ATP III Update $2004^{10}$.

Problemas auditivos y de visión fueron determinados mediante un cuestionario de tamizaje autorreportado $^{10}$.

Para depresión se utilizó el autorreporte de diagnóstico médico de depresión alguna vez en la vida, de la ENS 2009-2010 ${ }^{10}$.

\section{Análisis estadístico}

Para todos los análisis se utilizó el módulo de análisis de muestras complejas del programa Stata/ SE v15 y todos los resultados fueron estimados utilizando muestras expandidas según la ENS 2009-2010 ${ }^{10}$. Los datos de caracterización de la población estudiada se presentan como promedio y porcentaje expandidos con sus respectivos $95 \%$ intervalos de confianza (95\% IC) para variables continuas y categóricas, respectivamente.

Para determinar los principales factores asociados a sospecha de deterioro cognitivo (MMSE alterado) se realizaron análisis de regresión logística con varianza robusta para ajustar los modelos por la relación entre variables de exposición, covariables y la variable de salida, estos resultados fueron expresados como odds ratio y sus respectivos $95 \%$ IC. Las variables de exposición utilizadas fueron clasificadas en factores sociodemográficos, estilos de vida y condición de salud, las cuales han sido previamente identificadas en estudios internacionales $^{3,6,7}$. Para investigar si la asociación entre sospecha de deterioro cognitivo y las variables de exposición fue independiente de factores confundidores, se construyeron 3 modelos estadísticos: modelo 0: no ajustado, modelo 1: ajustado por sexo, edad, zona geográfica y nivel educacional y modelo 2: ajustado por el modelo 1 y por IMC y estilos de vida. El nivel de significancia fue definido como $\mathrm{p}<0,05$.

\section{Resultados}

Un 11,6\% [95\% IC: 8,8; 15,2] de los adultos mayores presentó sospecha de deterioro cognitivo, prevalencia que corresponde a un total de 277.899 adultos mayores con esta condición a nivel nacional.

En la Tabla 1 se reportan las características de la población según sospecha de deterioro cognitivo. En comparación a aquellos sin sospecha, la prevalencia de deterioro cognitivo incrementa con la edad y fue mayor en niveles bajos de escolaridad, zonas rurales, en personas viudas, jubiladas, físicamente inactivos, obesas y enflaquecidas, personas que duermen $>9 \mathrm{~h}$ y personas con una mala autopercepción de bienestar y salud. Las personas con sospecha de deterioro cognitivo también presentaron un menor peso corporal, nivel de actividad física y consumo de frutas y verduras, pero un mayor nivel de tiempo destinado a estar sentado. La prevalencia de sospecha de deterioro cognitivo fue mayor en personas con diabetes, hipertensión, problemas auditivos, discapacidad y depresión.

Los factores sociodemográficos asociados a sospecha de deterioro cognitivo fueron: $>8$ años de escolaridad (OR: 21,6, $\mathrm{p}<0,004)$, personas con 
Tabla 1. Características de la población mayor en Chile según sospecha de deterioro cognitivo

\begin{tabular}{|c|c|c|}
\hline \multirow[b]{2}{*}{ Variables } & \multicolumn{2}{|c|}{ Deterioro cognitivo } \\
\hline & Sin sospecha & Con sospecha \\
\hline \multicolumn{3}{|l|}{ Socio-demográficas } \\
\hline Participantes encuestados (n)* & $1.215(87,8)$ & $169(12,2)$ \\
\hline Muestra expandida $(n)^{*}$ & $2.112 .217(88,4)$ & $277.899(11,6)$ \\
\hline Prevalencia expandida de deterioro cognitivo, \% & $88,4(84,8 ; 91,2)$ & $11,6(8,8 ; 15,2)$ \\
\hline Edad (años) & $68,5(67,8 ; 69,2)$ & $76,3(73,9 ; 78,8)$ \\
\hline \multicolumn{3}{|l|}{ Grupos etarios, \% } \\
\hline 60-65 años & $97,1(94,6 ; 98,4)$ & $2,9(1,6 ; 5,3)$ \\
\hline 66-70 años & $87,5(75,3 ; 94,1)$ & $12,6(5,9 ; 24,6)$ \\
\hline 71-75 años & $93,8(88,8 ; 96,7)$ & $6,2(3,4 ; 11,2)$ \\
\hline 76-80 años & $77,6(62,4 ; 87,8)$ & $22,4(12,1 ; 37,5)$ \\
\hline$>80$ años & $63,7(51,7 ; 74,2)$ & $36,3(25,8 ; 48,3)$ \\
\hline \multicolumn{3}{|l|}{ Sexo, \% } \\
\hline Hombres & $86,5(79,3 ; 91,5)$ & $13,5(8,5 ; 20,7)$ \\
\hline Mujeres & $89,9(86,7 ; 92,4)$ & $10,1(7,6 ; 13,3)$ \\
\hline \multicolumn{3}{|l|}{ Zona geográfica, \% } \\
\hline Rural & $78,8(64,3 ; 88,5)$ & $21,2(11,5 ; 35,7)$ \\
\hline Urbano & $90,2(87,1 ; 92,7)$ & $9,8(7,3 ; 12,9)$ \\
\hline \multicolumn{3}{|l|}{ Estado civil, \% } \\
\hline Casado o con pareja & $89,1(83,6 ; 92,9)$ & $10,9(7,1 ; 16,4)$ \\
\hline Viudo & $83,5(76,4 ; 88,7)$ & $16,5(11,3 ; 23,6)$ \\
\hline Soltero & $92,3(85,1 ; 96,2)$ & $7,7(3,8 ; 14,9)$ \\
\hline \multicolumn{3}{|l|}{ Nivel educacional, \% } \\
\hline Básica (< 8 años) & $81,0(74,9 ; 85,9)$ & $19,0(14,1 ; 25,1)$ \\
\hline Media (8-12 años) & $95,3(91,4 ; 97,5)$ & $4,7(2,5 ; 8,6)$ \\
\hline Superior (> 12 años) & $99,0(95,9 ; 99,8)$ & $1,0(0,2 ; 4,1)$ \\
\hline \multicolumn{3}{|l|}{ Situación laboral, \% } \\
\hline Trabajador con ingresos & $94,9(90,5 ; 97,3)$ & $5,1(2,7 ; 9,5)$ \\
\hline Jubilado o rentista & $84,2(77,6 ; 89,1)$ & $15,8(10,9 ; 22,4)$ \\
\hline \multicolumn{3}{|l|}{ Antropométricas } \\
\hline Peso corporal (kg) & $71,7(70,2 ; 73,0)$ & $65,3(61,9 ; 68,3)$ \\
\hline Talla (m) & $1,58(1,57 ; 1,59)$ & $1,55(1,53 ; 1,57)$ \\
\hline $\mathrm{IMC}\left(\mathrm{kg} / \mathrm{m}^{2}\right)$ & $28,7(28,2 ; 29,3)$ & $27,3(25,9 ; 28,6)$ \\
\hline Perímetro cintura $(\mathrm{cm})$ & $95,8(94,6 ; 96,9)$ & $93,5(90,2 ; 96,9)$ \\
\hline \multicolumn{3}{|l|}{ Estado nutricional, \% } \\
\hline Bajo peso $\left(<23 \mathrm{~kg} / \mathrm{m}^{2}\right)$ & $77,2(63,6 ; 86,7)$ & $22,8(13,3 ; 36,4)$ \\
\hline Normal $\left(23,0-27,9 \mathrm{~kg} / \mathrm{m}^{2}\right)$ & $94,6(90,7 ; 97,0)$ & $5,4(3,0 ; 9,3)$ \\
\hline Sobrepeso $\left(28,0-31,9 \mathrm{~kg} / \mathrm{m}^{2}\right)$ & $89.8(84,9 ; 93,3)$ & $10,2(6,7 ; 15,1)$ \\
\hline Obeso $\left(\geq 32,0 \mathrm{~kg} / \mathrm{m}^{2}\right)$ & $82,7(71,6 ; 90,1)$ & $17,3(9,9 ; 28,4)$ \\
\hline \multicolumn{3}{|l|}{ Obesidad central, \% } \\
\hline Normal & $89,1(84,6 ; 92,4)$ & $10,9(7,6 ; 15,4)$ \\
\hline Obesidad & $87,7(81,9 ; 91,9)$ & $12,3(8,1 ; 18,1)$ \\
\hline
\end{tabular}




\begin{tabular}{|c|c|c|}
\hline \multicolumn{3}{|l|}{ Estilos de vida } \\
\hline Actividad física total (min/día) & $159,3(138,3 ; 180,3)$ & $74,0(40,3 ; 107,8)$ \\
\hline Tiempo sedentarismo (h/día) & $3,7(3,3 ; 4,1)$ & $5,5(3,8 ; 7,1)$ \\
\hline \multicolumn{3}{|l|}{ Inactividad física, \% } \\
\hline Activo & $93,7(90,8 ; 95,7)$ & $6,3(4,3 ; 9,2)$ \\
\hline Inactivo & $78,3(70,2 ; 85,3)$ & $21,3(14,7 ; 29,8)$ \\
\hline \multicolumn{3}{|l|}{ Categorías de sueño, \% } \\
\hline Normal (7-9 h/día) & $92,1(87,5 ; 95,2)$ & $7,9(4,8 ; 12,5)$ \\
\hline Bajo (< 7 h/día) & $91,3(87,3 ; 94,2)$ & $8,7(5,8 ; 12,7)$ \\
\hline Alto (> 9 h/día) & $73,9(61,7 ; 83,3)$ & $26,1(16,7 ; 38,3)$ \\
\hline Consumo de frutas y verduras (g/día) & $243,1(226,6 ; 259,5)$ & $196,4(165,8 ; 227,0)$ \\
\hline Consumo de sal (g/día) & $10,27(9,58 ; 10,68)$ & $10,80(9,56 ; 12,03)$ \\
\hline Consumo de alcohol (g/día) & $38,58(29,48 ; 49,69)$ & $42,59(24,69 ; 60,50)$ \\
\hline \multicolumn{3}{|l|}{ Estilos de vida saludable, \% } \\
\hline No saludable & $94,9(87,0 ; 98,1)$ & $5,1(1,9 ; 12,9)$ \\
\hline Moderadamente saludable & $88,6(84,2 ; 91,8)$ & $11,4(8,2 ; 15,7)$ \\
\hline Muy saludable & $73,5(55,9 ; 85,8)$ & $26,5(14,2 ; 44,1)$ \\
\hline \multicolumn{3}{|l|}{ Tabaquismo, \% } \\
\hline Nunca & $86,4(82,0 ; 89,9)$ & $13,6(10,1 ; 18,0)$ \\
\hline Ex-fumador & $87,3(78,4 ; 92,9)$ & $12,7(7,1 ; 21,6)$ \\
\hline Fumador & $95,2(90,0 ; 97,7)$ & $4,8(2,3 ; 10,0)$ \\
\hline \multicolumn{3}{|l|}{ Metabólicas y salud } \\
\hline \multicolumn{3}{|l|}{ Síndrome metabólico, \% } \\
\hline Normal & $89,3(82,4 ; 93,7)$ & $10,7(6,3 ; 17,6)$ \\
\hline Con síndorme metabólico & $90,8(85,9 ; 94,1)$ & $9,2(5,9 ; 14,1)$ \\
\hline \multicolumn{3}{|l|}{ Diabetes mellitus tipo 2, \% } \\
\hline Normal & $89,4(85,8 ; 92,2)$ & $10,6(7,8 ; 14,2)$ \\
\hline Con Diabetes mellitus tipo 2 & $85,5(74,1 ; 92,4)$ & $14,5(7,6 ; 25,9)$ \\
\hline \multicolumn{3}{|l|}{ Hipertensión arterial, \% } \\
\hline Sin hipertensión & $94,8(91,7 ; 96,8)$ & $5,1(3,2 ; 8,3)$ \\
\hline Con hipertensión & $82,2(75,5 ; 87,3)$ & $17.8(12,7 ; 24,4)$ \\
\hline \multicolumn{3}{|l|}{ Problemas auditivos, $\%$} \\
\hline No tiene problemas auditivos & $94,3(91,6 ; 96,1)$ & $5,7(3,9 ; 8,4)$ \\
\hline Si tiene problemas auditivos & $85,4(72,1 ; 93,0)$ & $14,6(7,0 ; 27,9)$ \\
\hline \multicolumn{3}{|l|}{ Calidad de la visión, \% } \\
\hline Buena & $95,5(92,8 ; 97,2)$ & $4,5(2,8 ; 7,2)$ \\
\hline Regular & $88,4(83,9 ; 91,7)$ & $11,6(8,3 ; 16,1)$ \\
\hline Mala & $71,2(57,6 ; 81,9)$ & $28,8(18,1 ; 42,4)$ \\
\hline \multicolumn{3}{|l|}{ Discapacidad, \% } \\
\hline Normal & $96,3(94,0 ; 97,8)$ & $3,7(2,2 ; 6,0)$ \\
\hline Con discapacidad & $77,1(69,8 ; 83,1)$ & $22,9(16,9 ; 30,2)$ \\
\hline \multicolumn{3}{|l|}{ Depresión, \% } \\
\hline Sin depresión & $89,4(85,1 ; 92,5)$ & $10,6(7,5 ; 14,9)$ \\
\hline Con depresión & $83,2(72,2 ; 90,5)$ & $16,8(9,5 ; 27,8)$ \\
\hline
\end{tabular}

Datos son presentados como promedio y desviación estándar para variables continuas, y como porcentaje para variables categóricas. *Todas las estimaciones fueron realizadas con el factor de expansión sugerido por la ENS2009-2010 para muestra nacional. La muestra encuestada corresponde al total de personas incluidas en la ENS 2009-2010, pero sin aplicar ningún factor de expansión. 
edades entre 76-80 y > 80 años (OR: 4,89 y 4,82, $\mathrm{p}<0,003$, respectivamente) y personas de sexo masculino (OR: 2,42, p = 0,015). No se encontró asociación entre deterioro cognitivo y zona de residencia, estado civil y situación laboral (Tabla 2, Figura 1).

Entre los estilos de vida asociados positivamente con sospecha de deterioro cognitivo se encuentras inactividad física (OR: 2,$07 ; \mathrm{p}=0,016)$, pasar más de 4 h sentado (OR: 2,$23 ; \mathrm{p}=0,011$ ), consumir $<5$ porciones/día de frutas y verduras (OR: $2,02, \mathrm{p}=0,048)$, dormir $>9 \mathrm{~h} /$ día (OR: $2,98, \mathrm{p}=0,013)$ y por último tener un puntaje moderado o no saludable para estilos de vida (OR: 2,95 y 6,10, p $<0,002$, respectivamente). No se encontró asociación entre deterioro cognitivo y consumo de alcohol, sal y tabaquismo (Tabla 3, Figura 1).

Personas con bajo peso (OR: $3,67, \mathrm{p}=0,008$ ), obesidad (OR: 3,32; $\mathrm{p}=0,026$ ), problemas de audición (OR: 2,26; $\mathrm{p}=0,016$ ), una baja calidad de visión (OR: 3,$89 ; \mathrm{p}=0,005)$, que han tenido depresión (OR: 3,$03 ; \mathrm{p}=0,011)$ y que presentan algún nivel de discapacidad (OR: 5,63; $\mathrm{p}<0,0001$ ), presentan una asociación positiva con sospecha de deterioro cognitivo. No se encontraron asociaciones para diabetes, síndrome metabólico, hipertensión arterial y obesidad central, después de ajustar los modelos por variables confundidoras (Tabla 4, Figura 1).

Tabla 2. Factores sociodemógraficos asociados a sospecha de deterioro cognitivo en personas mayores en Chile

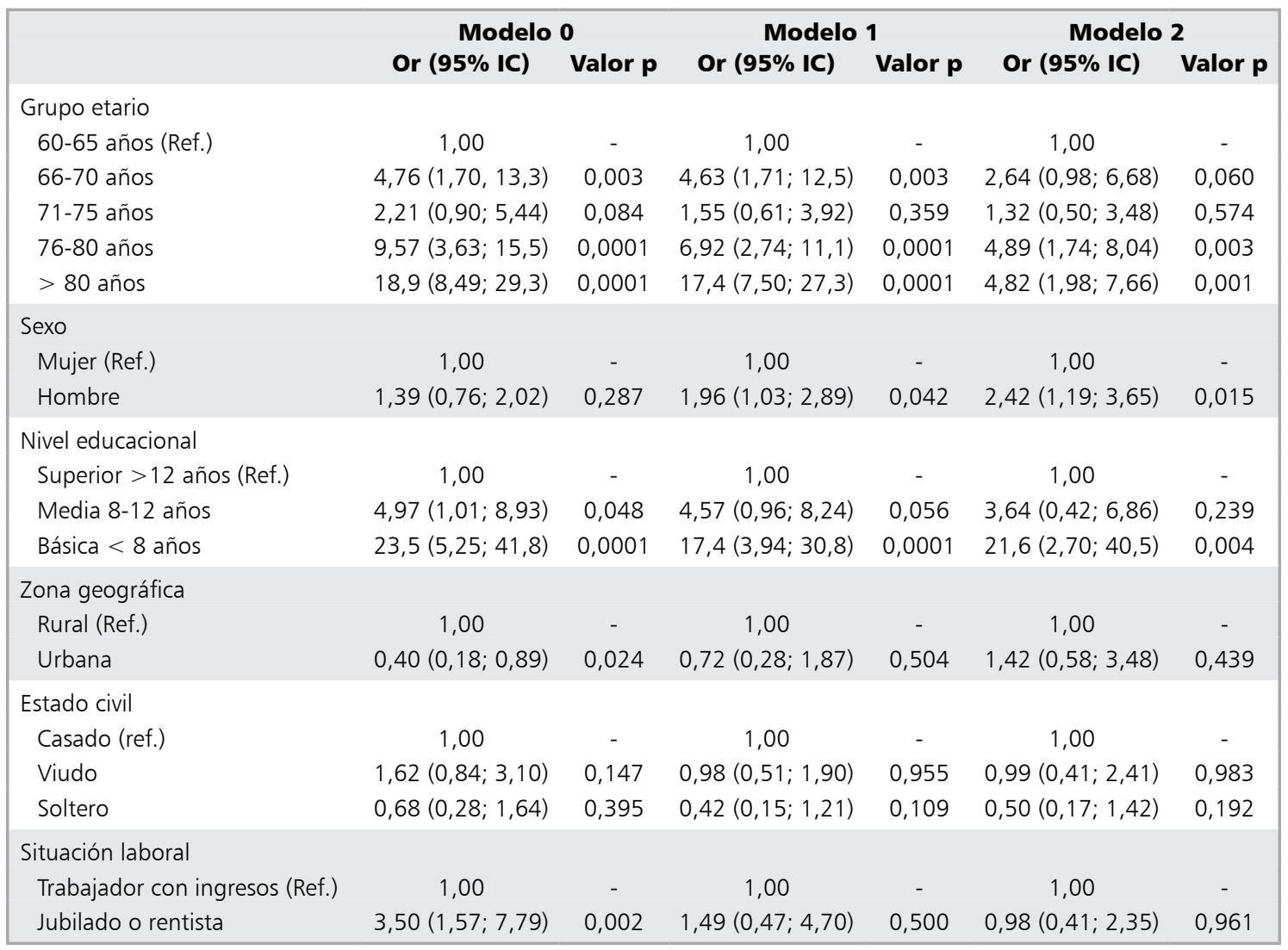

Datos presentados como odds ratio (OR) y sus respectivos $95 \%$ IC. Los análisis del modelo 0 no fueron ajustados por variables de confusión. El modelo 1 fue ajustado por edad, sexo, región, zona geográfica y nivel educacional. El modelo 2 fue ajustado por las variables del modelo 1 y adicionalmente por IMC e índice de estilos de vida saludable. Un OR $<1$ indica que hay una menor asociación entre la variable de exposición y sospecha de deterioro cognitivo mientras que un OR $>1$ indica que hay una mayor asociación con sospecha de deterioro cognitivo. 


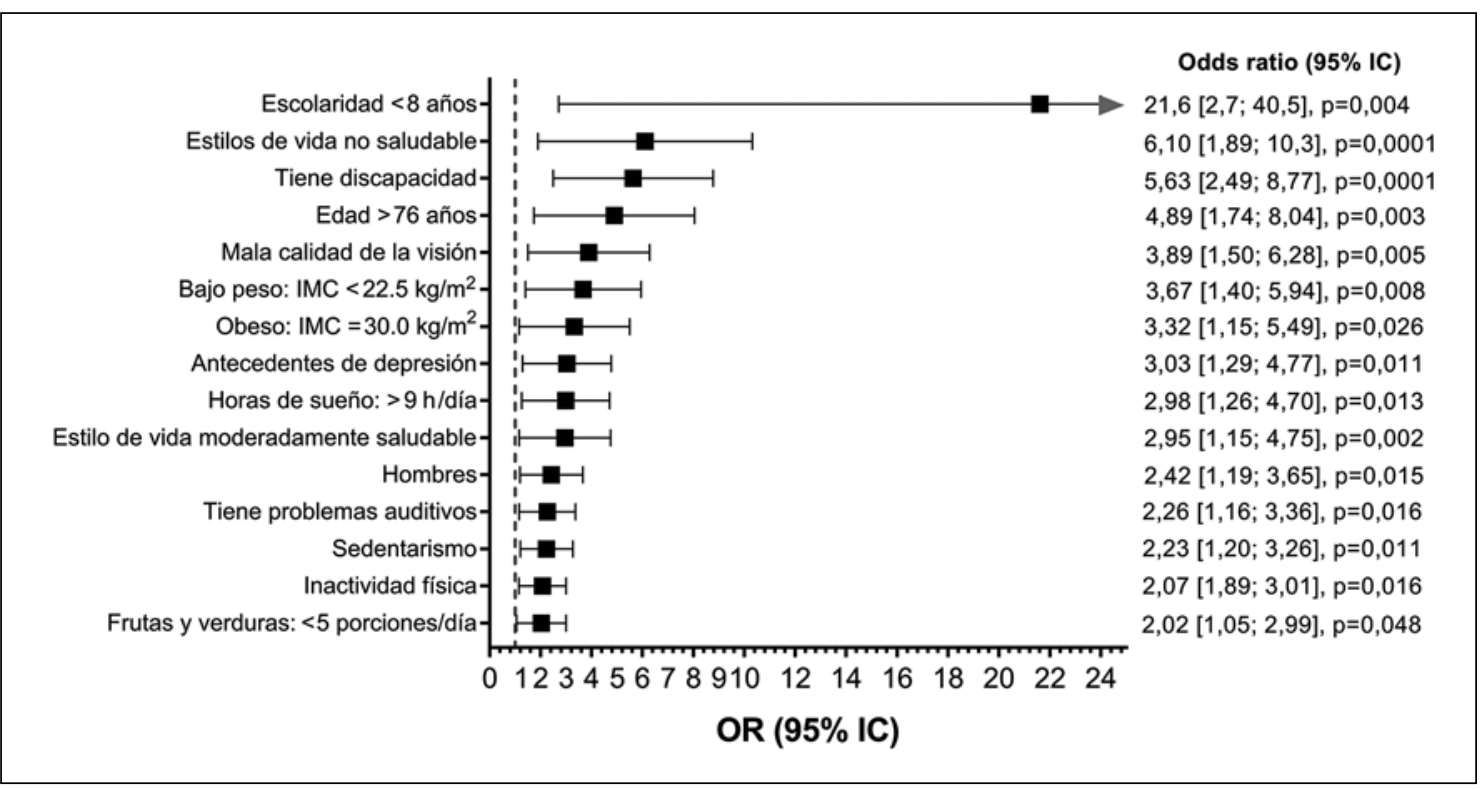

Figura 1. Factores asociados a sospecha de deterioro cognitivo en personas mayores en Chile. Los datos son presentados como odds ratio y sus $95 \%$ IC organizados según la magnitud de la asociación. Todas las estimaciones fueron generadas con el factor de expansión para población nacional. Los análisis fueron ajustado por el modelo 3 (edad, sexo, región, zona geográfica, nivel educacional, IMC e índice de estilos de vida saludable) a excepción de cuando estos factores fueron utilizados como variable independiente en los modelos estadísticos. Un $\mathrm{OR}<1$ indica que hay una menor asociación entre la variable de exposición y sospecha de deterioro cognitivo mientras que un $\mathrm{OR}>1$ indica que hay una mayor asociación con sospecha de deterioro cognitivo.

\section{Discusión}

Este estudio estimó que en promedio 1 de cada 10 personas mayores en Chile presenta sospecha de deterioro cognitivo, sin embargo, esta prevalencia aumenta drásticamente con la edad, alcanzando 3,0\% en población entre 60-65 años y $36,3 \%$ en personas mayores a 80 años. Los principales factores asociados a sospecha de deterioro cognitivo en población chilena fueron edad, bajo nivel de escolaridad, sexo masculino, inactividad física, alto tiempo sedente, bajo consumo de frutas y verduras, dormir $>9 \mathrm{~h} /$ día, malnutrición, tener problemas de audición o visuales, haber tenido depresión y tener alguna discapacidad. La identificación de estos factores de riesgo para sospecha de deterioro cognitivo podría ser de utilidad para la temprana pesquisa de personas con mayor probabilidad de sufrir esta condición, como también servir de base para el diseño de futuras intervenciones o programas de prevención orientados a mejorar estas conductas claves asociadas a sospecha de deterioro cognitivo.

Si bien este estudio incluyó 23 potenciales factores de riesgo que han sido asociados a sospecha de deterioro cognitivo en estudios internacionales ${ }^{1,3,6,7}$; solo 14 de ellos fueron asociados a sospecha de deterioro cognitivo en personas mayores en Chile (Figura 1). Los 3 factores con mayor asociación fueron bajo nivel de escolaridad $<8$ años, estilos de vida no saludable y tener discapacidad, mientras que los 3 factores con la menor magnitud de asociación fueron consumir $<5$ porciones de frutas y verduras al día, ser físicamente inactivo y sedentarismo (pasar sentado más de 4 h al día). Si bien Chile fue integrado recientemente a la lista de países de alto ingreso económico sus estilos de vida reflejan más bien una economía en transición que ha experimentado importantes cambios en sus estilos de vida en las últimas décadas ${ }^{17,18}$. Por este motivo, es importante poder corroborar si los factores de riesgo para sospecha de deterioro cognitivo reportados en otras poblaciones en el mundo son similares a los observados en Chile. 
Tabla 3. Estilos de vida asociados a sospecha de deterioro cognitivo en personas mayores en Chile

\begin{tabular}{|c|c|c|c|c|c|c|}
\hline & \multicolumn{2}{|c|}{ Modelo 0} & \multicolumn{2}{|c|}{ Modelo 1} & \multicolumn{2}{|c|}{ Modelo 2} \\
\hline & Or $(95 \%$ IC) & Valor p & Or (95\% IC) & Valor p & Or $(95 \%$ IC) & Valor $\mathbf{p}$ \\
\hline \multicolumn{7}{|l|}{ Actividad física } \\
\hline Activo (Ref.) & 1,00 & - & 1,00 & - & 1,00 & - \\
\hline Inactivo & $4,01(2,19 ; 5,83)$ & 0,000 & $2,91(1,45 ; 4,37)$ & 0,003 & $2,07(1,14 ; 3,00)$ & 0,016 \\
\hline \multicolumn{7}{|l|}{ Tiempo sedente } \\
\hline Bajo (< 4 h/día) & 1,00 & - & 1,00 & - & 1,00 & - \\
\hline Alto ( $\geq 4$ h/día) & $2,17(1,09 ; 2,66)$ & 0,027 & $1,68(1,08 ; 2,28)$ & 0,139 & $2,23(1,20 ; 3,26)$ & 0,011 \\
\hline \multicolumn{7}{|l|}{ Tabaquismo } \\
\hline Nunca (ref.) & 1,00 & - & 1,00 & - & 1,00 & - \\
\hline Ex fumador & $0,92(0,45 ; 1,90)$ & 0,830 & $1,21(0,59 ; 2,52)$ & 0,595 & $0,90(0,48 ; 1,72)$ & 0,755 \\
\hline Fumador & $0,32(0,14 ; 0,76)$ & 0,009 & $0,65(0,24 ; 1,76)$ & 0,393 & $0,65(0,22 ; 1,85)$ & 0,416 \\
\hline \multicolumn{7}{|l|}{ Consumo de alcohol } \\
\hline Bajo (< 7 g/día) & 1,00 & - & 1,00 & - & 1,00 & - \\
\hline Medio (7-44 g/día) & $1,40(0,36 ; 2,44)$ & 0,624 & $1,70(0,30 ; 3,10)$ & 0,546 & $2,15(0,31 ; 3,99)$ & 0,440 \\
\hline Alto (44 g/día) & $2,01(0,46 ; 3,56)$ & 0,356 & $2,18(0,41 ; 3,95)$ & 0,356 & $2,89(0,44 ; 5,34)$ & 0,268 \\
\hline \multicolumn{7}{|l|}{ Horas de sueño } \\
\hline Bajo (< 7 h/día) & $1,11(0,56 ; 1,66)$ & 0,761 & $1,18(0,61 ; 1,75)$ & 0,622 & $1,16(0,60 ; 1,72)$ & 0,655 \\
\hline Normal (7-9 h/día) (Ref) & 1,00 & - & 1,00 & - & 1,00 & - \\
\hline Alto (> 9 h/día) & $4,13(1,93 ; 6,33)$ & 0,0001 & $3,20(1,32 ; 5,08)$ & 0,010 & $2,98(1,26 ; 4,70)$ & 0,013 \\
\hline \multicolumn{7}{|l|}{ Consumo de frutas y verduras } \\
\hline 5 o más porciones/día (ref.) & 1,00 & - & 1,00 & - & 1,00 & - \\
\hline$<5$ porciones/día & $1,99(1,03 ; 2,95)$ & 0,045 & $1,84(1,02 ; 2,66)$ & 0,046 & $2,02(1,05 ; 2,99)$ & 0,048 \\
\hline \multicolumn{7}{|l|}{ Consumo de sal } \\
\hline$<9$ g/día & 1,00 & - & 1,00 & - & 1,00 & - \\
\hline$>9$ g/día & $1,09(0,50 ; 2,41)$ & 0,827 & $1,34(0,58 ; 3,08)$ & 0,488 & $1,04(0,46 ; 2,31)$ & 0,930 \\
\hline \multicolumn{7}{|l|}{ Estilo de vida } \\
\hline Muy saludable (ref.) & 1,00 & - & 1,00 & - & 1,00 & - \\
\hline Moderadamente saludable & $2,38(1,06 ; 3,70)$ & 0,014 & $3,12(1,16 ; 5,08)$ & 0,023 & $2,95(1,15 ; 4,75)$ & 0,002 \\
\hline No saludable & $6,66(1,85 ; 11,4)$ & 0,010 & $5,69(1,51 ; 9,87)$ & 0,010 & $6,10(1,89 ; 10,3)$ & 0,0001 \\
\hline
\end{tabular}

Datos presentados como odds ratio (OR) y sus respectivos $95 \%$ IC. Los análisis del modelo 0 no fueron ajustados por variables de confusión. El modelo 1 fue ajustado por edad, sexo, región, zona geográfica y nivel educacional. El modelo 2 fue ajustado por las variables del modelo 1 y adicionalmente por IMC. Un OR $<1$ indica que hay una menor asociación entre la variable de exposición y sospecha de deterioro cognitivo mientras que un $\mathrm{OR}>1$ indica que hay una mayor asociación con sospecha de deterioro cognitivo.

Nuestros resultados confirman en gran parte estos factores de riesgo, por lo cual las estrategias de prevención de deterioro cognitivo sugeridas por organizaciones internacionales de salud también serían aplicables a población adulta mayor en Chile ${ }^{5}$.

Entre los factores que no mostraron asociación en nuestro estudio, pero que si han sido establecidos como factores de riesgo en reportes internacionales, se encuentra el estado civil, y situación laboral, ya que personas viudas o jubiladas presentan un mayor riesgo se deterioro cognitivo, el cual ha sido vinculado a un proceso de reducción de la interacción con otras personas ${ }^{3,5}$. Otro factor que ha presentado una fuerte asociación con deterioro cognitivo es el tabaquismo, sin embargo, nuestro estudio no encontró asociación, esto podría explicarse por la carencia de datos más específicos tales como cantidad, duración y frecuencia de hábitos tabáquicos ${ }^{3,5}$. Similarmente, el consumo de alcohol ha sido reportado como un fuerte factor de riesgo para deterioro cognitivo, si bien nuestros resultados van en la misma dirección estos no fueron estadísticamente significativo, lo 
Tabla 4. Condiciones de salud asociadas a sospecha de deterioro cognitivo en personas mayores en Chile

\begin{tabular}{|c|c|c|c|c|c|c|}
\hline & \multicolumn{2}{|c|}{ Modelo 0} & \multicolumn{2}{|c|}{ Modelo 1} & \multicolumn{2}{|c|}{ Modelo 2} \\
\hline & Or (95\% IC) & Valor p & Or $(95 \%$ IC) & Valor p & Or $(95 \%$ IC) & Valor $p$ \\
\hline \multicolumn{7}{|l|}{ Estado nutricional } \\
\hline Bajo peso & $5,22(2,15 ; 8,29)$ & 0,0001 & $3,49(1,42 ; 5,56)$ & 0,007 & $3,67(1,40 ; 5,94)$ & 0,008 \\
\hline Normal (Ref.) & 1,00 & - & 1,00 & - & 1,00 & - \\
\hline Sobrepeso & $2,00(0,95 ; 4,20)$ & 0,066 & $1,93(0,89 ; 4,20)$ & 0,097 & $1,36(0,57 ; 3,25)$ & 0,487 \\
\hline Obeso & $3,68(1,54 ; 5,82)$ & 0,003 & $3,87(1,44 ; 6,30)$ & 0,008 & $3,32(1,15 ; 5,49)$ & 0,026 \\
\hline \multicolumn{7}{|l|}{ Obesidad central } \\
\hline Normal (Ref.) & 1,00 & - & 1,00 & - & 1,00 & - \\
\hline Obeso central & $1,14(0,62 ; 2,09)$ & 0,669 & $1,08(0,55 ; 2,14)$ & 0,823 & $1,12(0,48 ; 2,58)$ & 0,795 \\
\hline \multicolumn{7}{|l|}{ Salud auditiva } \\
\hline No tiene problema auditivo (Ref). & 1,00 & - & 1,00 & - & 1,00 & - \\
\hline $\begin{array}{l}\text { Tiene } 1 \text { o más problemas } \\
\text { auditivos }\end{array}$ & $3,56(2,00 ; 5,12)$ & 0,0001 & $2,67(1,45 ; 3,89)$ & 0,002 & $2,26(1,16 ; 3,36)$ & 0,016 \\
\hline \multicolumn{7}{|l|}{ Calidad de la visión } \\
\hline Buena (Ref). & 1,00 & - & 1,00 & - & 1,00 & - \\
\hline Regular & $2,81(1,50 ; 5,27)$ & 0,001 & $2,03(1,04 ; 3,96)$ & 0,038 & $1,87(0,83 ; 4,22)$ & 0,129 \\
\hline Mala & $8,60(3,93 ; 13,2)$ & 0,0001 & $5,36(2,26 ; 8,46)$ & 0,0001 & $3,89(1,50 ; 6,28)$ & 0,005 \\
\hline \multicolumn{7}{|l|}{ Diabetes } \\
\hline No (ref.) & 1,00 & - & 1,00 & - & 1,00 & - \\
\hline Sí & $1,43(0,64 ; 3,17)$ & 0,380 & $1,82(0,78 ; 4,27)$ & 0,166 & $1,74(0,71 ; 4,23)$ & 0,224 \\
\hline \multicolumn{7}{|l|}{ Hipertensión } \\
\hline No (ref.) & 1,00 & - & 1,00 & - & 1,00 & - \\
\hline Sí & $3,13(1,68,5,83)$ & 0,0001 & $1,85(0,94 ; 3,60)$ & 0,071 & $1,72(0,86 ; 3,49)$ & 0,127 \\
\hline \multicolumn{7}{|l|}{ Síndrome metabólico } \\
\hline No (ref.) & 1,00 & - & 1,00 & - & 1,00 & - \\
\hline $\mathrm{Si}$ & $0,85(0,40 ; 1,82)$ & 0,678 & $0,80(0,35 ; 1,85)$ & 0,605 & $0,61(0,27 ; 1,38)$ & 0,233 \\
\hline \multicolumn{7}{|l|}{ Antecedentes de depresión } \\
\hline No (ref.) & 1,00 & - & 1,00 & - & 1,00 & - \\
\hline Si & $1,69(0,80 ; 2,58)$ & 0,170 & $2,80(1,35 ; 4,25)$ & 0,006 & $3,03(1,29 ; 4,77)$ & 0,011 \\
\hline \multicolumn{7}{|l|}{ Discapacidad } \\
\hline Sin discapacidad (Ref.) & 1,00 & - & 1,00 & - & 1,00 & - \\
\hline Con discapacidad & $7,81(4,09 ; 11,5)$ & 0,0001 & $6,47(3,08 ; 9,86)$ & 0,0001 & $5,63(2,49 ; 8,77)$ & 0,0001 \\
\hline
\end{tabular}

Datos presentados como odds ratio (OR) y sus respectivos $95 \%$ IC. Los análisis del modelo 0 no fueron ajustados por variables de confusión. El modelo 1 fue ajustado por edad, sexo, región, zona geográfica y nivel educacional. El modelo 2 fue ajustado por las variables del modelo 1 y adicionalmente por IMC e índice de estilos de vida saludable. Un OR $<1$ indica que hay una menor asociación entre la variable de exposición y sospecha de deterioro cognitivo mientras que un OR $>1$ indica que hay una mayor asociación con sospecha de deterioro cognitivo.

cual podría estar explicado por el tamaño muestral de nuestro estudio, pero también por la falta de datos que permitan clasificar el tipo de alcohol consumido ${ }^{3,5,19}$. Otros factores que han mostrado asociación con deterior cognitivo es la presencia de factores de riesgo cardiovascular, como diabetes e hipertensión arterial, sin embargo, nuestro estudio no pudo replicar estas asociaciones, que en parte podrían ser explicadas por el efecto de mediación del IMC, ya que nuestros modelos fueron ajustado por este marcador de obesidad, el cual podría estar mediando la asociación entre deterioro cognitivo y factores de riesgo cardiovascular ${ }^{3,5,12}$.

Entre las fortalezas de este estudio se encuentra el análisis de datos de la ENS 2009-2010, con representatividad nacional y regional, lo que permitió 
establecer los principales factores relacionados con sospecha de deterioro cognitivo en personas mayores en Chile ${ }^{10}$. Sin embargo, dichos resultados deben ser considerados tomando en cuenta las limitaciones propias de un estudio de tipo transversal, que no permite establecer relaciones de causalidad. Otro factor por considerar es la sensibilidad y especificidad de la prueba MINI-MENTAL, la cual ha mostrado una moderada precisión para la identificación de deterioro cognitivo ${ }^{20,21}$. Además, debemos considerar el sesgo asociado al reclutamiento, ya que personas con algún nivel más severo de deterioro cognitivo pudieron haber sido excluidas de la ENS. Adicionalmente, el uso de medidas de autorreporte está sujeta a eventual sesgo en la precisión de la información recolectada, lo cual podría influir en la magnitud de las asociaciones encontradas.

En conclusión, este estudio identificó 14 factores de riesgo para sospecha de deterioro cognitivo, entre los cuales se destacan factores de riesgo tradicionales como la edad, sexo, nivel de escolaridad, alimentación, inactividad física, y malnutrición, pero también factores de riesgo emergentes, como discapacidad, problemas visuales y de audición, tiempo que destinamos a estar sentados y las horas destinadas a dormir. Los resultados de este estudio podrían ser de utilidad para identificar tempranamente grupos de riesgo para sospecha de deterioro cognitivo, y así aplicar más efectivamente iniciativas de intervención orientadas a prevenir o retrasar la aparición de deterioro cognitivo y su posible evolución hacia otras patologías neurodegenerativas como la demencia en personas mayores en Chile.

Agradecimientos: Se agradece de manera especial a todos los participantes de la ENS 200910, como también así al equipo profesional del Departamento de Salud Pública de la Facultad de Medicina de la Pontificia Universidad Católica de Chile y al Ministerio de Salud del Gobierno de Chile, quienes desarrollaron y aplicaron la Encuesta Nacional de Salud 2009-2010.

\section{Referencias}

1. WHO. Dementia http://www.who.int: World Health Organization; 2017. Available: http://www.who.int/ mediacentre/factsheets/fs362/en/
2. Brookmeyer R, Johnson E, Ziegler-Graham K, Arrighi HM. Forecasting the global burden of Alzheimer's disease. Alzheimers Dement 2007; 3 (3): 186-91. doi: 10.1016/j.jalz.2007.04.381.

3. Livingston G, Sommerlad A, Orgeta V, Costafreda SG, Huntley J, Ames D, et al. Dementia prevention, intervention, and care. Lancet 2017; 390 (10113): 2673-734. doi: 10.1016/S0140-6736(17)31363-6.

4. MINSAL. Plan Nacional de Demencia 2017. Santiago, Chile: MINSAL 2017. Available: https://www.minsal.cl/ wp-content/uploads/2017/11/PLAN-DE-DEMENCIA. pdf.

5. WHO. Risk reduction of cognitive decline and dementia: WHO Guidelines. Geneva, Switzerland: World Health Organization, (ALC) DoAaL-C; 2019. Available: https://www.who.int/ageing/en/.

6. WHO. World report on ageing and health. Geneva: World Health Organization, 2015. Available: https:// www.who.int/ageing/publications/world-report-2015/ en/.

7. Plassman BL, Williams JW, Jr., Burke JR, Holsinger T, Benjamin S. Systematic Review: Factors Associated With Risk for and Possible Prevention of Cognitive Decline in Later Life. Ann Intern Med 2010; 153 (3): 182-93. doi: 10.7326/0003-4819-153-3-201008030-00258.

8. MINSAL. Encuesta Nacional de Consumo de Alimentos. Santiago, Chile: MINSAL, Medicina Fd; 2014. Available: https://www.minsal.cl/sites/default/files/ENCA-INFORME_FINAL.pdf.

9. Troncoso C, Petermann-Rocha F, Brown R, Maria Leiva A, Adela Martinez M, Diaz-Martinez X, et al. Patterns of healthy lifestyle behaviours in older adults: Findings from the Chilean National Health Survey 2009-2010. Exp Gerontol. 2018;113:180-5. doi: 10.1016/j.exger.2018.09.026.

10. MINSAL. Encuesta Nacional de Salud 2009-2010. Chile: MINSAL, 2010. Available: http://web.minsal.cl/portal/ url/item/bcb03d7bc28b64dfe040010165012d23.pdf.

11. WHO. Global Health and Aging 2011. World Health Organization; US National Institute of Aging, 2011. October 2011. Report No.: Contract No.: 06 June. Available: http://www.who.int/ageing/publications/ global_health.pdf.

12. Petermann F, Troncoso-Pantoja C, Martínez MA, Leiva AM, Ramírez-Campillo R, Poblete-Valderrama F, et al. Risk of cognitive impairment among older people with diabetes or family history of the disease. Rev Med Chile 2018; 146 (8): 872-81. doi: 10.4067/s003498872018000800872.

13. Cancino M, Rehbein-Felmer L, Ortiz MS. Funcionamiento cognitivo en adultos mayores: rol de la reserva 
cognitiva, apoyo social y depresión. Rev Med Chile 2018; 146: 315-22.

14. Donoso A, Venegas P, Villarroel C, Vásquez C. Deterioro cognitivo leve y enfermedad de Alzheimer inicial en adultos mayores. Rev Chil Neuro-Psiquiatr. 2001; 39 (3): 231-8. doi: http://dx.doi.org/10.4067/S071792272001000300007.

15. García-Hermoso A, Ramírez-Velez R, Celis-Morales $\mathrm{CA}$, Olloquequi J, Izquierdo M. Can physical activity attenuate the negative association between sitting time and cognitive function among older adults? A mediation analysis. Exp Gerontol 2018; 106: 173-7. doi: 10.1016/j. exger.2018.03.002.

16. Celis-Morales C, Salas C, Alduhishy A, Sanzana R, Martínez MA, Leiva A, et al. Socio-demographic patterns of physical activity and sedentary behaviour in Chile: results from the National Health Survey 20092010. J Public Health (Oxf) 2016; 38 (2): e98-e105. doi: 10.1093/pubmed/fdv079.

17. Vio F, Albala C, Kain J. Nutrition transition in Chile revisited: mid-term evaluation of obesity goals for the period 2000-2010. Public Health Nutr 2008; 11 (4): 405-12. doi: 10.1017/s136898000700050x.

18. Barria RM, Amigo H. Nutrition transition: a review of Latin American profile. Arch Latinoam Nutr 2006; 56 (1): 3-11.

19. Sabia S, Fayosse A, Dumurgier J, Dugravot A, Akbaraly $\mathrm{T}$, Britton A, et al. Alcohol consumption and risk of dementia: 23 year follow-up of Whitehall II cohort study. BMJ. 2018;362. doi: https://doi.org/10.1136/ bmj.k2927.

20. Carnero Pardo C, Cruz Orduña I, Espejo Martínez B, Cárdenas Viedma S, Torrero García P, Olazarán Rodríguez J. Efectividad del Mini-Mental en la detección del deterioro cognitivo en Atención Primaria. Atención Primaria 2013; 45 (8): 426-33. doi: 10.1016/j. aprim.2013.04.009.

21. Quiroga LP, Albala BC, Klaasen PG. Validación de un test de tamizaje para el diagnóstico de demencia asociada a edad, en Chile. Rev Med Chile 2004; 132: 467-78. doi: http://dx.doi.org/10.4067/S003498872004000400009. 